\title{
Relationship between Human Eye and Different Divergence of Skeletal Class I Pattern: A Correlative Study
}

\author{
Garima Gupta ${ }^{1}$, Shashank Gupta ${ }^{2}$, Manish Goyal ${ }^{3}$, Neha Gupta ${ }^{4}$
}

\begin{abstract}
Introduction: All the systems in the body are interconnected to form a single structural unit. Scientific evaluations of potential correlations between the stomatognathic system and the eye are based on different scientific approaches. The development of the eye and the stomatognathic system begins at approximately the same time, that is, during the $3 \mathrm{rd}$ and 4 th week of intrauterine life. Some of the recent studies have shown that the various genes and growth factors involved in the eye formation play an important role in the development of the stomatognathic system also. Aims and objectives: The aim of the present study is to correlate the normal human eye variables with the different growth patterns. Materials and methods: A total of 150 samples were selected. The sample selected for the study ranged in the age group from 18 to 24 years. Individual were subjected to radiographs (lateral cephalograms) and various eye tests. The subjects were divided into horizontal, average, and vertical growth patterns on the basis of the Go-Gn-SN angle.

Results: Positive correlation was found between visual acuity and mandibular plane angle among the average growth pattern, and negative correlation was found between visual acuity and mandibular plane angle among the horizontal growth pattern. So, the present study concludes that there is a correlation between the human eye variables and the growth patterns, and thus malocclusion can be anticipated on this basis.. Keywords: Divergence, Eye, Pax-6, Pax-9, Skeletal pattern.

Journal of Mahatma Gandhi University of Medical Sciences \& Technology (2018): 10.5005/jp-journals-10057-0073
\end{abstract}

\section{INTRODUCTION}

All the systems in the body are interconnected to form a single structural unit. Scientific evaluations of potential correlations between the stomatognathic system and the eye are based on different scientific approaches. Some recent studies have shown that the various genes and growth factors involved in the eye formation play an important role in the development of the stomatognathic system also., ${ }^{1,2}$

In this study, patterns of horizontal, average, or vertical growth were diagnosed from the lateral cephalograms. These growth patterns were correlated with the visual acuity, corneal diameter, corneal thickness, axial length, retinal nerve fiber layer thickness, and intraocular pressure of each patient.

\section{Materials and Methods}

The 150 samples were selected. Good-quality lateral cephalograms of 150 patients were obtained based on the following criteria:

\section{Inclusion Criteria}

- Subjects of age group 18-24 years having a class I skeletal pattern

- Subjects should not have any facial deformity or syndrome

\section{Exclusion Criteria}

- Subjects having class II and class III skeletal pattern

- Subjects with history of orthodontic, orthognathic, or plastic surgery

- Subjects with history of eye surgery

\section{Sampling Method}

The subjects were divided into three groups: ${ }^{3}$

- Group I-Horizontal growth pattern Go Gn-SN angle = 25-29
1Department of Orthodontics and Dentofacial Orthopedics, Gupta Dental and Implant Center, Jaipur, Rajasthan, India

${ }^{2}$ Department of Oral Medicine and Radiology, Mahatma Gandhi Dental College and Hospital, Mahatma Gandhi Uninversity of Medical Sciences and Technology, Jaipur, Rajasthan, India

${ }^{3}$ Department of Orthodontics and Dentofacial Orthopedics, Teerthanker Mahaveer University, Moradabad, Uttar Pradesh, India ${ }^{4}$ Department of Pathology, SMS Medical College, Rajasthan, Jaipur, India

Corresponding Author: Shashank Gupta, Department of Oral Medicine and Radiology, Mahatma Gandhi Dental College and Hospital, Mahatma Gandhi Uninversity of Medical Sciences and Technology, Jaipur, Rajasthan, India, Phone: +91 9571717788, e-mail: dentist.shashank@gmail.com

How to cite this article: Gupta G, Gupta S, Goyal M, et al. Relationship between Human Eye and Different Divergence of Skeletal Class I Pattern: A Correlative Study. J Mahatma Gandhi Univ Med Sci Tech 2018;3(2): 54-60.

Source of support: Nil

Conflict of interest: None

- Group II-Average growth pattern Go Gn-SN angle = 30-34

- Group III-Vertical growth pattern Go Gn-SN angle = 35-39

\section{Methodology}

The standardized lateral cephalogram of each patient was obtained. The cephalometric landmarks were identified: angle between point A-Nasion-point $B(A N B)$, beta angle, and mandibular plane angle.

The subjects were then referred to a renowned eye institute with academic interest. Different eye test variables were established and identified by a qualified ophthalmologist.

Eye test variables taken were the following:

(c) The Author(s). 20180pen Access This article is distributed under the terms of the Creative Commons Attribution 4.0 International License (https://creativecommons. org/licenses/by-nc/4.0/), which permits unrestricted use, distribution, and non-commercial reproduction in any medium, provided you give appropriate credit to the original author(s) and the source, provide a link to the Creative Commons license, and indicate if changes were made. The Creative Commons Public Domain Dedication waiver (http://creativecommons.org/publicdomain/zero/1.0/) applies to the data made available in this article, unless otherwise stated. 
Visual Acuity ${ }^{4}$

During an eye test, ophthalmologists use eye charts to measure how well you see in the distance, compared with other human beings. The standard placement of the eye chart is on a wall that is 20 feet away from your eyes. Vision is considered "normal" when there is a 20/20 vision (or 20/20 visual acuity), that is, a letter can be read at 20 feet.

\section{Corneal Diameter ${ }^{5}$}

The corneal diameter (CD) is the limbus-to-limbus distance, and clinically both the horizontal and vertical dimensions are regarded as important.

\section{Corneal Thickness ${ }^{6}$}

Corneal thickness measurements are indicative of the metabolic status of the cornea, as they provide an index of corneal hydration. Such measurements give valuable information on the physiological status of the cornea and its changes associated with disease, trauma, and hypoxia.

\section{Axial Length ${ }^{7}$}

The axial length is the distance from the corneal surface to an interference peak corresponding to the retinal pigment epithelium. A majority of axial length elongation takes place in the first 36 months of life and a gradual reduction of growth over the next 2 years, and by 3 years the adult size is attained.

\section{Retinal Nerve Fiber Layer (RNFL) ${ }^{8}$}

Optical coherence tomography is a relatively new noncontact imaging technique that has been developed to assess tissue thickness with micrometer scale sensitivity.

\section{Intraoccular Pressure (IOP) ${ }^{7}$}

In human eyes in particular, the axial length undergoes daily fluctuations of some $15-40 \mu \mathrm{m}$, with a mean period of approximately 21 hours. Although diurnal fluctuations in axial length have been observed in many species, the underlying physiologic control mechanisms are unknown.

Various scans obtained during investigation were shown in Figure 1 and interpreted in Figure 2.

\section{Statistical Analysis}

The data obtained were subjected to statistical analysis.

The statistical analysis was done using Statistical Package of Social Science. Data comparison was done by applying specific statistical tests to find out the statistical significance of the comparisons. Quantitative variables were compared using mean values and qualitative variables using proportions. Significance level was fixed at $p<0.05$.

\section{Results}

Table 1 illustrates descriptive statistics for optical parameters for group I. The mean corneal diameter of the left eye was slightly higher than the right. The mean corneal thickness of the right eye was slightly higher than the left. The mean axial length of the right eye was slightly higher than the left. The mean RNLF of the right eye was slightly higher than the left. The mean IOP of the right eye was slightly higher than the left.

Table 2 illustrates descriptive statistics for optical parameters for group II. The mean corneal diameter of the left eye was slightly higher than the right. The mean corneal thickness of the right eye was slightly higher than the left. The mean axial length of the right eye was slightly higher than the left. The mean RNLF of the right
Table 1: Descriptive statistics for optical parameters for group

\begin{tabular}{lcccc}
\hline & & & & \multicolumn{1}{l}{$\begin{array}{l}\text { Std. } \\
\text { deviation }\end{array}$} \\
\hline Visual acuity RT & 0.0000 & 0.0000 & 0.000000 & 0.0000000 \\
Visual acuity LT & 0.0000 & 0.1760 & 0.017600 & 0.0533361 \\
Corneal diameter & 10.90 & 12.69 & 11.8468 & 0.48110 \\
RT & & & & \\
Corneal diameter & 10.81 & 12.80 & 12.0356 & 0.48401 \\
LT & & & & \\
Corneal thickness & 450 & 604 & 537.88 & 52.057 \\
RT & & & & \\
Corneal thickness & 454 & 598 & 537.12 & 47.709 \\
LT & & & & \\
Axial length RT & 21.63 & 24.55 & 23.3376 & 0.70960 \\
Axial length LT & 21.00 & 24.30 & 23.3194 & 0.86634 \\
RNLF RT & 78 & 110 & 100.34 & 8.270 \\
RNLF LT & 79 & 111 & 100.32 & 8.326 \\
IOP RT & 10 & 18 & 14.36 & 2.238 \\
IOP LT & 10 & 17 & 13.66 & 2.309 \\
\hline
\end{tabular}

Table 2: Descriptive statistics for optical parameters for group II

\begin{tabular}{lcccc}
\hline & & & & \multicolumn{1}{l}{$\begin{array}{l}\text { Std. } \\
\text { deviation }\end{array}$} \\
\hline Visual acuity RT & 0.0000 & 0.3010 & 0.016580 & 0.0588661 \\
Visual acuity LT & 0.0000 & 0.3979 & 0.022039 & 0.0725589 \\
Corneal diameter & 11.00 & 13.58 & 11.9902 & 0.50838 \\
RT & & & & \\
Corneal diameter & 11.00 & 13.00 & 12.0948 & 0.47594 \\
LT & & & & \\
Corneal thickness & 450 & 608 & 539.00 & 41.528 \\
RT & & & & \\
Corneal thickness & 452 & 611 & 536.14 & 41.750 \\
LT & & & & \\
Axial length RT & 21.81 & 24.83 & 23.5388 & 0.79004 \\
Axial length LT & 21.00 & 24.80 & 23.5346 & 0.84260 \\
RNLF RT & 78 & 110 & 97.74 & 9.120 \\
RNLF LT & 80 & 110 & 97.32 & 9.410 \\
IOP RT & 12 & 18 & 13.94 & 2.045 \\
IOP LT & 10 & 18 & 13.26 & 2.146 \\
\hline & & & & \\
\hline
\end{tabular}

Table 3: Descriptive statistics for optical parameters for group III

\begin{tabular}{lcccc}
\hline & & & & \multicolumn{2}{l}{$\begin{array}{l}\text { Std. } \\
\text { deviation }\end{array}$} \\
\hline Visual acuity RT & 0.0000 & 0.5440 & 0.036295 & 0.1019236 \\
Visual acuity LT & 0.0000 & 0.6990 & 0.043047 & 0.1112065 \\
Corneal diameter & 10.99 & 13.58 & 11.8400 & 0.53919 \\
RT & & & & \\
Corneal dia LT & 11.00 & 12.70 & 11.8708 & 0.37218 \\
Corneal thickness & 454 & 590 & 535.98 & 30.178 \\
RT & & & & \\
Corneal thickness & 456 & 590 & 536.12 & 31.277 \\
LT & & & & \\
Axial length RT & 21.73 & 25.10 & 23.5351 & 0.77267 \\
Axial length LT & 21.50 & 25.15 & 23.5139 & 0.85975 \\
RNLF RT & 85 & 111 & 95.53 & 8.352 \\
RNLF LT & 83 & 115 & 93.08 & 7.958 \\
IOP RT & 10 & 18 & 13.41 & 1.779 \\
IOP LT & 10 & 16 & 12.88 & 1.752 \\
\hline
\end{tabular}




\begin{tabular}{lllllll} 
Name: & BHARDWAJ, RAGINI & & OD & OS & & ZEISS \\
ID: & P00012 & Exam Date: & $6 / 11 / 2016$ & $6 / 11 / 2016$ & CL Gupta Eye Institute & \\
DOB: & $6 / 11 / 1993$ & Exam Time: & $5: 23 \mathrm{PM}$ & $5: 26 \mathrm{PM}$ & \\
Gender: & Female & Technician: & Cirrus Operator. & \\
Doctor: & & Signal Strength: & $3 / 10 \quad 3 / 10$ & & \\
\hline
\end{tabular}

\section{\begin{tabular}{ll|l}
\hline RNFL Thickness Analysis:Optic Disc Cube 200x200 OD & $\bigcirc$ OS \\
\hline
\end{tabular}}

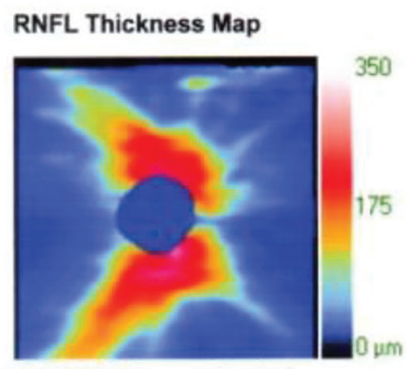

RNFL Thickness Deviation

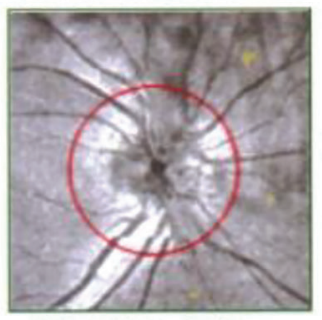

Offset $(-0.15 ;-0.18) \mathrm{mm}$ RNFL TSNIT Normative Data

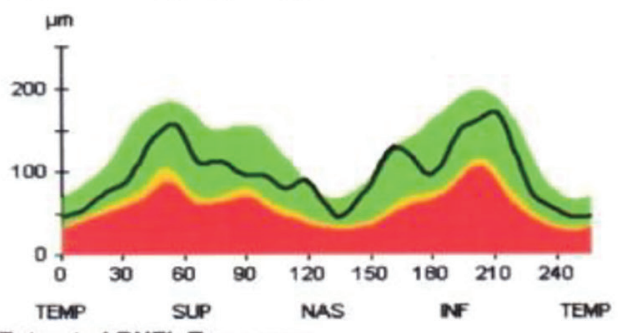

Extracted RNFL Tomogram

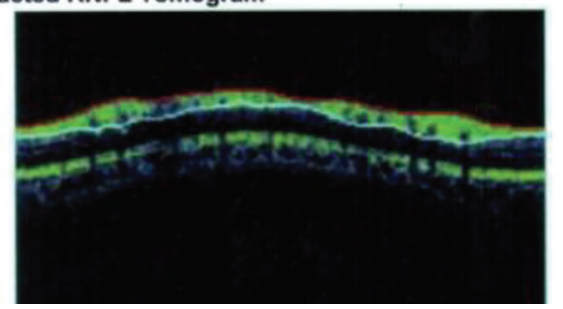

Fig. 1: Scan

eye was slightly higher than the left. The mean IOP of the right eye was slightly higher than the left.

Table 3 illustrates descriptive statistics for optical parameters for group III. The mean corneal diameter of the left eye was slightly higher than the right. The mean corneal thickness of the left eye was slightly higher than the right. The mean axial length of the right eye was slightly higher than the left. The mean RNLF of the right eye was slightly higher than the left. The mean IOP of the right eye was slightly higher than the left.

Table 4 illustrates the Pearson correlation matrix between dental and optical parameters among group I, which shows negative correlation between visual acuity of the left eye and mandibular plane angle, beta angle, and ANB angle.

Table 5 illustrates the Pearson correlation matrix between dental and optical parameters among group II, which shows positive correlation between visual acuity of the left eye and mandibular plane angle.

Table 6 illustrates the Pearson correlation matrix between dental and optical parameters among group III, which shows no statistically significant correlations between dental and optical parameter correlation to be found. Although there was statistically significant correlation was found within the optical as well as dental parameters. 


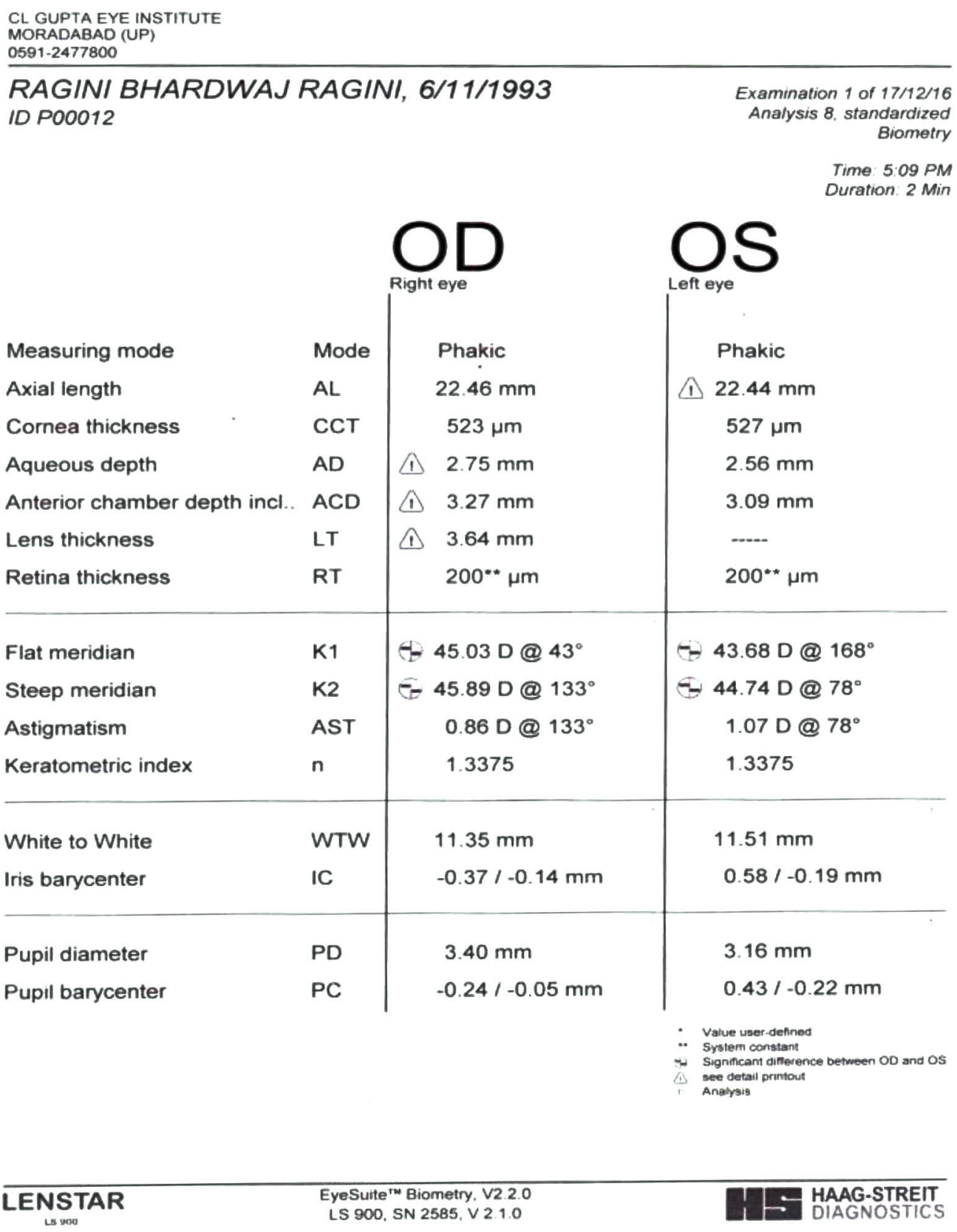

Fig. 2: Interpretation of scan

\section{Discussion}

Eye development is initiated by the master control gene Pax-6, a homeobox gene, and so is the stomatognathic system. The Pax- 6 gene locus is a transcription factor for the various genes and growth factors involved in eye formation. ${ }^{2,9}$

In an immunohistochemical study by Lei, the expression of Pax- 6 was seen in various developmental stages of the tooth. At the bud stage and cap stage, Pax- 6 was expressed in the central parts of the tooth buds/enamel organs and in the oral epithelia adjacent to the tooth germs.'

As the various systems in the body are interconnected to form a single structural unit, a pathological condition in one area can also affect other areas. There are many known correlations between the visual and motor system. The importance of visual function, particularly the paracentral peripheral field of view, in motor coordination, ambulation, and the maintenance of balance has been amply demonstrated. In line with current medical principles, which are moving toward a more holistic view of the human body, this study aims to investigate, in an interdisciplinary manner, the incidence of dental malocclusions together with posture and eye convergence disorders. This study was conducted to determine the clinical association between teeth malocclusions, wrong posture, and ocular convergence disorders. A total of 605 children attending at the 3rd, 4th, and 5 th years of seven Genoa primary schools were examined. Each child underwent the following examinations: (i) dental/occlusal, 


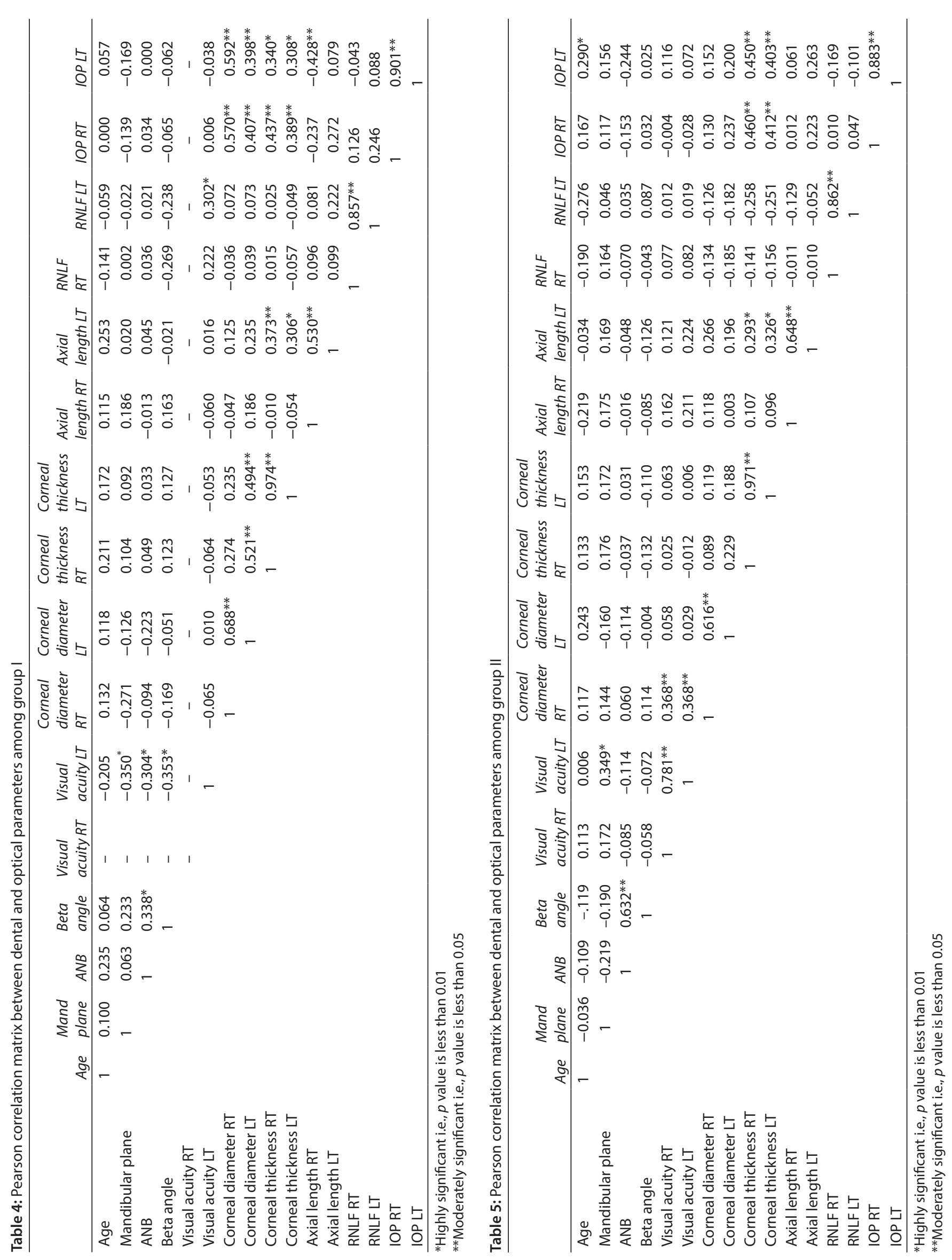


(ii) orthoptic, and (iii) postural. Occlusal data concerned the presence of cross-bite, midline deviation with a mandibular shift, bad habits, and deep or open bite. Postural assessment involved frontal and lateral inspection, investigation during trunk flexion and ambulation, and note of any asymmetry in the lower limbs. The recorded orthoptic data included those pertaining to ocular dominance, a cover test, convergence, and the Brock string test. A prevalence of cases with an unphysiological gait was found in patients with overjet (14.70\%) or overbite $(14.87 \%)$, while the percentage of patients with normal occlusion who showed an unphysiological gait was $13.08 \%$. Also, about 93.8 to $94.2 \%$ of children showed normal legs without dysmetry, with no difference in respect to the type of occlusion. Subjects with an open bite or deep bite showed a slightly different distribution of right or left dominant eyes. About $13 \%$ of children showed a pathological gait and, among them, vertical anomalies of occlusion (deep bite or open bite) were prevalent with respect to the other occlusal defects. The vertical dimension of occlusion revealed a slight relationship with the proper dominant eye. Postural, orthoptic, osteopathic, and occlusal variables were often clinically associated, and therefore these disorders appear to request a multidisciplinary medical approach for their treatment. $^{10}$

Lin et al. conducted a study at Tufts University, School of Dental Medicine, and confirmed the relationship between head posture, mandibular position, and eye dominance. The purpose of this study is to assess the temporary eye dominance of children and the change relative to head posture and mandibular position. Twenty children were included in this study after the completion of the ophthalmological exclusion screening. All subjects had the sign of eye dominance screened with eye dominance tests. The mandibular position and the three-dimensional posture of head were recorded. The eye patches were applied over the dominant eye for 1 hour to achieve the effect of temporary eye dominance change for 10 children. Ten children had the same procedures except for the eye patch procedure. The reexaminations were performed at the end of 1-hour visit for both groups. Mandibular midline data revealed no change in both control and experimental groups. The transverse head postural change has been demonstrated by means of temporary eye dominance change. This study highlights several areas: the importance of clinical intervention in young children, the difference on transverse dimensional change, and the importance of the role of pediatric dentistry among interdisciplinary cooperation. Further investigation and early clinical intervention are encouraged. ${ }^{11}$

In the present study, the skeletal class I growth pattern was assessed using the beta angle, and horizontal, average, or vertical growth patterns were diagnosed from the lateral cephalograms. These growth patterns were correlated with the visual acuity, corneal diameter, corneal thickness, axial length, retinal nerve fiber layer thickness, and intraocular pressure of each patient.

\section{Correlation between the Dental and Ophthalmic Parameters}

\section{Group I: Horizontal Grower}

A negative correlation is seen between the visual acuity of the left eye and the mandibular plane in group I, i.e., horizontal grower. By looking at the above correlation, we can anticipate some malocclusion in this type of grower but the type of malocclusion cannot be determined as we have not taken dental malocclusion 
into consideration in our study. Thus, the visual acuity can serve as a basis for the determination of malocclusion in a horizontal grower.

\section{Group II: Average Grower}

A positive correlation is seen between the visual acuity of the left eye and the mandibular plane in group II, i.e., average grower. It was observed that by determining the visual acuity in an average grower, it can be assumed that some type of malocclusion may be present.

\section{Group III: Vertical Grower}

There was no statistically significant correlations between dental and optical parameters in group III. However, there was a statistically significant correlation that was found within the optical as well as dental parameters.

\section{Conclusion}

Positive correlation was found between visual acuity and mandibular plane angle among the average growth pattern, and negative correlation was found between visual acuity and mandibular plane angle among the horizontal growth pattern. So, correlation is present between dental and eye variables.

Hence, if the patient is referred from an ophthalmologist, then by analyzing the various eye variables and correlating them with the growth pattern of the patient, malocclusion can be forestalled.

In the future, it will serve as an excellent interdisciplinary approach to diagnosis and treatment planning.

\section{References}

1. Simpson TI, Price DJ. Pax6; a pleiotropic player in development. Bioessays 2002;24(11):1041-1051. DOI: 10.1002/bies.10174.

2. Lei $\mathrm{H}$, Liu H, et al. Immunohistochemical localization of Pax6 in the developing tooth germ of mice. J Mol Histol 2014;45(4):373-379. DOI: 10.1007/s10735-014-9564-5.

3. Grover N, Kapoor DN, et al. Smile analysis in different facial patterns and its correlation with underlying hard tissues. Prog Orthod 2015;16(1):28. DOI: 10.1186/s40510-015-0099-4.

4. Wu PC, Chen YJ, et al. Assessment of macular retinal thickness and volume in normal eyes and highly myopic eyes with third-generation optical coherence tomography. Eye 2008;22(4):551-555. DOI: 10.1038/ sj.eye.6702789.

5. Mashige KP. A review of corneal diameter, curvature and thickness values and influencing factors. African Vision and Eye Health 2013;72(4):185-194. DOI: 10.4102/aveh.v72i4.58.

6. Eballe AO, Koki G, et al. Central corneal thickness and intraocular pressure in the Cameroonian nonglaucomatous population. Clin Ophthalmol 2010;4:717. DOI: 10.2147/OPTH.S10575.

7. Wilson $L B, Q u i n n G E$, et al. The relation of axial length and intraocular pressure fluctuations in human eyes. Invest Ophthalmol Vis Sci 2006;47(5):1778-1784. DOI: 10.1167/iovs.05-0869.

8. Paunescu LA, Schuman JS, et al. Reproducibility of nerve fiber thickness, macular thickness, and optic nerve head measurements using Stratus OCT. Invest Ophthalmol Vis Sci 2004;45(6):1716-1724. DOI: 10.1167/iovs.03-0514.

9. Sadler TW. Langman's Med embryology. Lippincott Williams \& Wilkins; 2011 Dec 15.

10. Silvestrini-Biavati A, Migliorati M, et al. Clinical association between teeth malocclusions, wrong posture and ocular convergence disorders: an epidemiological investigation on primary school children. BMC pediatrics 2013;13(1):12. DOI: 10.1186/1471-2431-13-12.

11. Lin SY, White GE. Mandibular position and head posture as a function of eye dominance. J Clin Pediat Dent 1996;20(2):133-140. 